\title{
Nuove applicazioni della medicina nucleare in ambito diagnostico: II parte
}

Recent applications of nuclear medicine in diagnostics: II part

\author{
G. Treglia,a* E. Cason, ${ }^{\mathrm{b}}$ G. Fagiolib \\ aIstituto di Medicina Nucleare, Policlinico “A. Gemelli”, Università Cattolica del Sacro Cuore, \\ Roma \\ bUO Medicina Nucleare, Ospedale "Maggiore”, ASL di Bologna
}

\begin{abstract}
*Corresponding author: Istituto di Medicina Nucleare, Policlinico “A. Gemelli”, Università Cattolica del Sacro Cuore, l.go Gemelli 8 - 00168 Roma.
\end{abstract}

Received 5 January 2010

Accepted 13 January 2010

Available online 11 June 2010

http://www.sciencedirect.com/science/article/pii/S1877934410000800

\begin{abstract}
Introduction

Positron-emission tomography (PET) and single photon emission computed tomography (SPECT) are effective diagnostic imaging tools in several clinical settings. The aim of this article (the second of a 2-part series) is to examine some of the more recent applications of nuclear medicine imaging techniques, particularly in the fields of neurology, cardiology, and infection/inflammation.
\end{abstract}


Discussion

A review of the literature reveals that in the field of neurology nuclear medicine techniques are most widely used to investigate cognitive deficits and dementia (particularly those associated with Alzheimer disease), epilepsy, and movement disorders. In cardiology, SPECT and PET also play important roles in the work-up of patients with coronary artery disease, providing accurate information on the state of the myocardium (perfusion, metabolism, and innervation). White blood cell scintigraphy and FDG-PET are widely used to investigate many infectious/inflammatory processes. In each of these areas, the review discusses the use of recently developed radiopharmaceuticals, the growth of tomographic nuclear medicine techniques, and the ways in which these advances are improving molecular imaging of biologic processes at the cellular level.

\section{Keywords}

Nuclear medicine; Cardiology; Neurology; Inflammatory states; Imaging. 